\title{
Evaluation of differential qPE9-1/DEP1 protein domains in rice grain length and weight variation
}

\author{
Xiangbo Li', Quandan Tao', Jun Miao', Zefeng Yang ${ }^{1,2}$, Minghong Gu ${ }^{1,2}$, Guohua Liang ${ }^{1,2^{*}}$ (D) and Yong Zhou ${ }^{1,2^{*}}$
}

\begin{abstract}
Background: $9 P E 9-1 / D E P 1$, encoding a $\mathrm{G}$ protein $\gamma$ subunit, has multiple effects on plant architecture, grain size, and yield in rice. The qPE9-1 protein contains an N-terminal G gamma-like (GGL) domain, a putative transmembrane domain, and a C-terminal cysteine-rich domain. However, the roles of each domain remain unclear.

Results: In the present study, we focused on the genetic effects of different domains of qPE9-1 in the regulation of grain length and weight. We generated a series of transgenic plants expressing different truncated qPE9-1 proteins through constitutive expression and clustered regularly interspaced palindromic repeats (CRISPR)/CRISPRassociated protein 9 strategies. Phenotypic analysis indicated that the complete or long-tailed qPE9-1 contributed to the elongation of grains, while the GGL domain alone and short-tailed qPE9-1 led to short grains. The long Cterminus of qPE9-1 including two or three C-terminal von Willebrand factor type $\mathrm{C}$ domains effectively repressed the negative effects of the GGL domain on grain length and weight. qPE9-1-overexpressing lines in a Wuxianggeng 9 (carrying a qpe9-1 allele) background showed increased grain yield per plant, but lodging occurred in some years.

Conclusions: Manipulation of the C-terminal length of qPE9-1 through genetic engineering can be used to generate varieties with various grain lengths and weights according to different requirements in rice breeding. The genetic effects of qPE9-1/ape9-1 are multidimensional, and breeders should take into account other factors including genetic backgrounds and planting conditions in the use of qPE9-1/ape9-1.
\end{abstract}

Keywords: Rice, Heterotrimeric G protein, Grain size, Yield production

\section{Background}

As one of the most important crops worldwide, rice (Oryza sativa L.) provides $35 \%-60 \%$ of the world's dietary calories and is consumed by more than 3 billion people (Fageria, 2007). During the last half-century, global rice production has increased dramatically, primarily because of genetic improvements resulting from the use of semi-dwarfing genes and heterosis to produce hybrid rice. However, an apparent plateau in development has been observed in the last 20 years (Yang and Zhang, 2010).

\footnotetext{
*Correspondence: ricegb@yzu.edu.cn; zhouyong@yzu.edu.cn 'Jiangsu Key Laboratory of Crop Genetics and Physiology / Key Laboratory of Plant Functional Genomics of the Ministry of Education / Jiangsu Key Laboratory of Crop Genomics and Molecular Breeding, Agricultural College of Yangzhou University, Yangzhou 225009, China

Full list of author information is available at the end of the article
}

Grain length not only influences rice grain yield but also affects the physical appearance of the grain and the quality of rice for cooking and eating. Rice grains range from less than $3 \mathrm{~mm}$ to more than $11 \mathrm{~mm}$ in length (Fitzgerald et al., 2009). The preference for rice varieties with different grain lengths varies among consumer groups (Li et al., 2004). For instance, varieties with long, slender grains are preferred by consumers and cultivators in the USA and most Asian countries. However, short, round grain varieties are popular in Japan, South Korea, and Sri Lanka (Li et al., 2004). Thus, rice grain length has a direct effect on marketability and, hence, commercial success. Grain length also affects 1000-grain weight, one of the yield components of rice, and subsequently determines rice productivity (Tan et al., 2000). To date, more than 60 genes determining rice grain length and weight have been cloned, and several 
signaling pathways including heterotrimeric $\mathrm{G}$ protein, the ubiquitin-proteasome pathway, the mitogen-activated protein kinase signaling pathway, phytohormones and transcriptional regulatory factors, have been investigated (Li et al., 2016b; Xu et al., 2016; Li et al., 2018).

G proteins consisting of $G_{\alpha}, G_{\beta}$, and $G_{\gamma}$ subunits are involved in a wide range of plant processes including morphological development (Fujisawa et al., 1999; Utsunomiya et al., 2011; Li et al., 2012; Thung et al., 2012; Peng et al., 2018), cell proliferation (Ullah et al., 2001; Chen et al., 2003; Ishida et al., 2014), stomatal control (Wang et al., 2015), abiotic stress (Ma et al., 2015; Yu and Assmann, 2015; Kaur et al., 2018), ion channel regulation (Chakravorty et al., 2011), light perception and protection (Ferrero-Serrano et al., 2018), and responses to phytohormones (Shi et al., 2015; Subramaniam et al., 2016; Zhang et al., 2017). Humans possess $23 G_{\alpha}$, five $G_{\beta}$, and $12 G_{\gamma}$ subunits (Wettschureck and Offermanns, $2005)$, while the rice genome has only one $\mathrm{G}_{\alpha}(R G A 1)$, one $\mathrm{G}_{\beta}(R G B 1)$, and five $\mathrm{G}_{\beta}$ (RGG1, RGG2, GS3, qPE91 , and GGC2) genes (Sun et al., 2018). Rice dwarf mutant $d 1$, which is defective in $R G A 1$, showed defective gibberellin signal transduction and produces small grains (Ashikari et al., 1999; Fujisawa et al., 1999; Ueguchi-Tanaka et al., 2000). RGB1 knockdown lines had decreased plant heights, and reduced panicle and grain sizes compared with wild-type (Utsunomiya et al., 2011). Thus, $R G A 1$ and $R G B 1$ are positive regulators in rice grain size. However, the $G_{\gamma}$ subunit-encoding genes play diverse roles in grain development.

GS3 was the first molecularly characterized quantitative trait locus (QTL) for grain size in rice (Fan et al., 2006). Varieties containing a $\mathrm{C} \rightarrow \mathrm{A}$ natural mutation in the second exon resulting in loss of the GS3 allele produce extremely long grains. The GS3 protein is composed of a plant-specific organ size regulation (OSR) domain in the $\mathrm{N}$-terminus, a transmembrane domain, a tumor necrosis factor receptor/nerve growth factor receptor (TNFR/NGFR) family cysteine-rich domain, and a von Willebrand factor type C (VWFC) domain in the $\mathrm{C}$ terminus, which function differentially in grain size regulation (Fan et al., 2006; Mao et al., 2010). The OSR domain (also considered a G gamma-like domain, GGL) is both necessary and sufficient to limit grain size, whereas C-terminal TNFR/NGFR and VWFC domains have an inhibitory effect on the OSR function. Deletion of the C-terminal cysteine-rich region leaving most of the OSR domain intact causes plants to produce extremely short grains (Mao et al., 2010).

Previously, we characterized a major rice QTL associated with plant and panicle architecture, $q P E 9-1$ (Zhou et al., 2009), which is allelic to DEP1 (Huang et al., 2009). Deletion of part of the C-terminal region at this locus reduced the plant height, and produced small grains and short, erect panicles; the mutation allele, qpe 9-1, is widely employed in most japonica varieties in China. $q P E 9-1 / D E P 1$ was also shown to be involved in nitrogen use efficiency in rice (Sun et al., 2014), while we detected a function for $q P E 9-1$ as a negative regulator in abscisic acid (ABA)-dependent drought-stress responses (Zhang et al., 2015). These data indicate that qPE9-1/DEP1 has multiple effects on rice growth and development, and plays essential roles in yield production.

The qPE9-1 protein contains an N-terminal GGL domain, a putative transmembrane domain, and a C-terminal cysteine-rich domain. To investigate the effects of these domains on grain size regulation, we herein generated a series of transgenic plants expressing different truncated qPE9-1 proteins through constitutive expression and CRISPR/Cas9 (clustered regularly interspaced short palindromic repeats and CRISPR-associated protein 9) strategies.

\section{Results}

qPE9-1 acts as a positive regulator of grain size in rice CRISPR/Cas9 technology has been demonstrated to achieve efficient targeted mutagenesis in transgenic rice (Hu et al., 2016, Ma et al., 2016; Li et al., 2017; Lu et al., 2017), so was used here to specifically disrupt $q P E 9-1$. We designed single guide (sg) RNAs targeting the second and fifth exon of $q P E 9-1$, and simultaneously transformed them into Nipponbare. More than 20 independent transgenic lines were obtained. Sequencing of PCR-amplified qPE9-1 genomic DNA from the transgenic plants resulted in the identification of at least 12 independent and effective knockout mutants. Three independent homozygous mutant lines, qpe9-1\#1, qpe9-1\#2, and qpe9-1\#3, were confirmed and grown for agronomic trait examination.

The mutation sites of qpe9-1\#1 and qpe9-1\#2 occurred at the second exon, which generated two knockout mutants of $q P E 9-1$ (Fig. 1). These two mutants lost most of the qPE9-1 protein, which covered part of the GGL domain and all C-terminal VWFC domains (Fig. 2a, b). The average grain lengths of qpe9-1\#1 and qpe9-1\#2 were $7.15 \pm 0.14$ and $7.14 \pm 0.16 \mathrm{~mm}$, respectively, while the wild-type grain length was $7.47 \pm 0.24 \mathrm{~mm}$. Thus, qpe9$1 \# 1$ and qpe $9-1 \# 2$ plants showed an average $4.37 \%$ reduction in grain length (Fig. 2c, d). The 1000-grain weight of qpe9-1\#1 and qpe9-1\#2 plants was significantly decreased by $6.17 \%$ and $6.48 \%$, respectively (Fig. $2 \mathrm{e}$ ). The mutation site in qpe9-1\#3 lines was located in the fifth exon of the target gene, and caused a deletion of the qPE9-1 $\mathrm{C}$-terminal (Fig. 2a, b). The grain length and weight of qpe $9-1 \# 3$ were significantly decreased by $5.08 \%$ and $9.52 \%$, respectively (Fig. $2 \mathrm{~d}, \mathrm{e}$ ). These data show that intact qPE9-1 acts as a positive regulator of grain length and weight in rice, and that knockout of $q P E 9-1$ leads to short and small grains. 


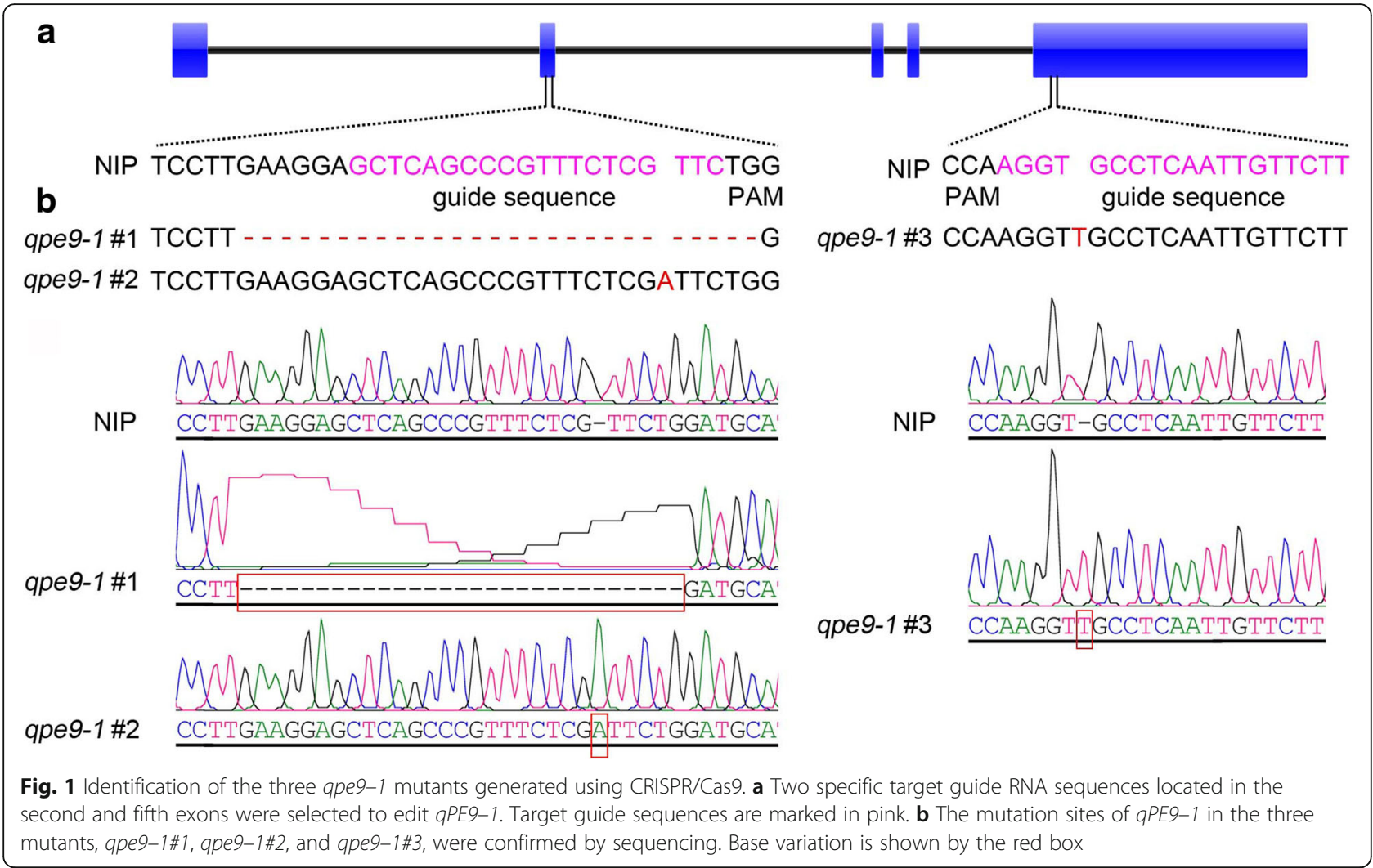

\section{Overexpression of different QPE9-1 protein domains generates grains of different length and weight}

To further uncover the function of each domain, several constructs covering different domains of qPE9-1 were generated in which the coding sequences were driven by the maize ubiquitin promoter and then delivered to Nipponbare. We developed a set of transgenic plants overexpressing full-length qPE9-1 (FL), truncated qPE9-1 without all three VWFC domains (D1), truncated qPE91 without the second and third VWFC domains (D2), truncated qPE9-1 without the third VWFC domain (D3), and truncated qPE9-1 without part of the C-terminus (D4) (Fig. 3a). At least eight independent transgenic individuals for each construct were obtained. All $\mathrm{T}_{1}$ families were grown and screened with single-copy transferred DNA insertion using hygromycin resistance as the marker. Seeds from a single $T_{1}$ plant were harvested, then transgenic lines of the $\mathrm{T}_{2}$ generation were grown, and homozygous lines (namely, D1-OE, D2-OE, D3-OE, D4-OE, and FL-OE) for each construct were selected for phenotypic observation.

As shown in Fig. 3b, the transgenic plants overexpressing the full-length cDNA sequence of $q P E 9-1$ produced larger and heavier grains than wild-type. Compared with Nipponbare, the grain length and weight of FL-OE transgenic plants increased by $11.36 \%$ and $14.44 \%$, respectively (Fig. 3c, d). These results further confirm that
qPE9-1 is a positive regulator of grain length and weight in rice. D4-OE plants also produced enlarged grains (+ $11.96 \%$ in grain length and $+12.71 \%$ in 1000 -grain weight) which were similar to those of FL-OE transgenic plants. However, overexpression of the D3 construct had only a mild effect on grain size increase $(6.13 \%$ in grain length and $7.88 \%$ in 1000 -grain weight) (Fig. $3 \mathrm{~b}$ ).

The transformants overexpressing the truncated qPE9-1 protein lacking the second and third VWFC domains produced smaller grains (Fig. $3 \mathrm{~b}$ ). We observed a substantial decrease in grain length $(-5.99 \%)$ and 1000 -grain weight $(-13.70 \%)$ in D2-OE transgenic plants compared with those of Nipponbare (Fig. 3c, d). Moreover, transgenic plants carrying the D1 construct overexpressing the GGL domain also exhibited reduced grain length $(-6.97 \%)$ and weight $(-13.17 \%)$ (Fig. 3). This suggested that the N-terminal GGL domain of qPE9-1 negatively regulates grain length and weight, and that C-terminal VWFC domains may inhibit the effects of the $\mathrm{N}$-terminal to promote grain development.

Constitutive expression of QPE9-1 results in higher grain yield per plant in an existing high-yield variety

1000-grain weight is one of the determinants of grain yield in rice. In view of the large effects of $q P E 9-1$ on grain length and weight, we wondered whether qPE9-1 could be used to further increase grain yield in an 

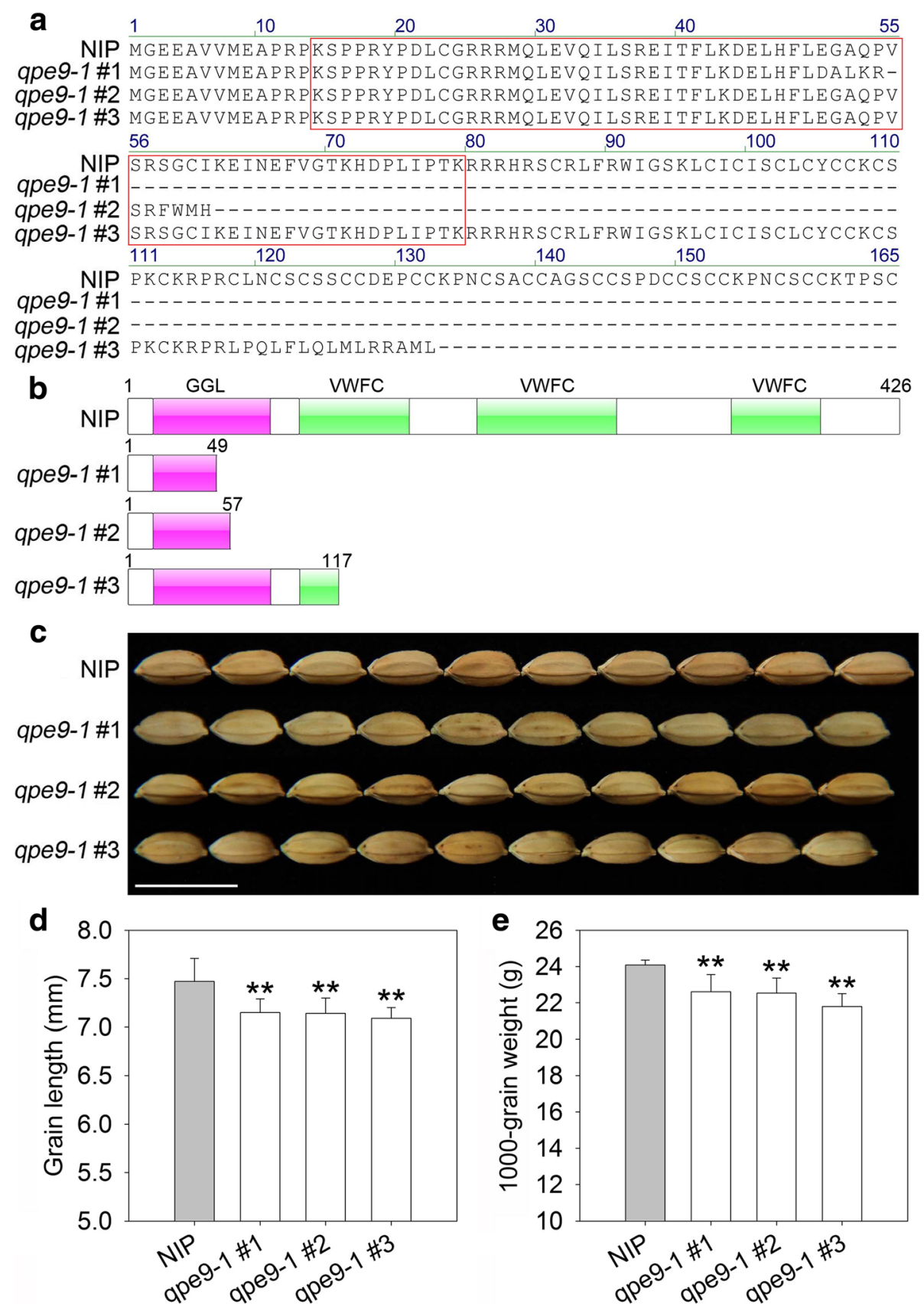

Fig. 2 Gene editing of qPE9-1 led to small grains in the Nipponbare background. a Protein sequences of qPE9-1 in the NIP, ape9-1\#1, qpe9-1\#2, and qpe9-1\#3 plants. The GGL domain is shown by red boxes. b Structure of the qPE9-1 protein in NIP, qpe9-1\#1, qpe9-1\#2, and qpe9-1\#3 plants. The numbers represent the different lengths of the truncated qPE9-1 protein in wild-type plants and the three mutants. c Grains of wild-type plants and the three ape9-1 mutants. Bar $=1 \mathrm{~cm}$. $\mathbf{d}$ Comparison of grain length between NIP and the three qpe9-1 mutants. e Comparison of 1000-grain weight between NIP and the three qpe9-1 mutants. Data are shown as means \pm SDs $(n=15)$. Student's $t$-test: $* * 0.01$

existing high-yield variety. A construct containing full-length $q P E 9-1$ cDNA driven by the maize ubiquitin promoter was transformed into Wuxianggeng 9 (WXG9, carrying a qpe9-1 allele), a high-yield fragrant variety of rice, which was widely cultivated in the south of Jiangsu Province, China. More than 20 independent transgenic lines were obtained, and three $\mathrm{T}_{8}$ generation homozygous lines (WXG9-OE1, WXG9-OE2, and WXG9-OE3,) were grown for a detailed phenotypic examination.

qPCR indicated that $q P E 9-1$ expression levels were significantly elevated in overexpressing lines compared with wild-type (Fig. 4c). At maturity, the three qPE91-overexpressing lines were significantly taller than wild-type (Fig. 4d). WXG9-OE1, WXG9-OE2, and 


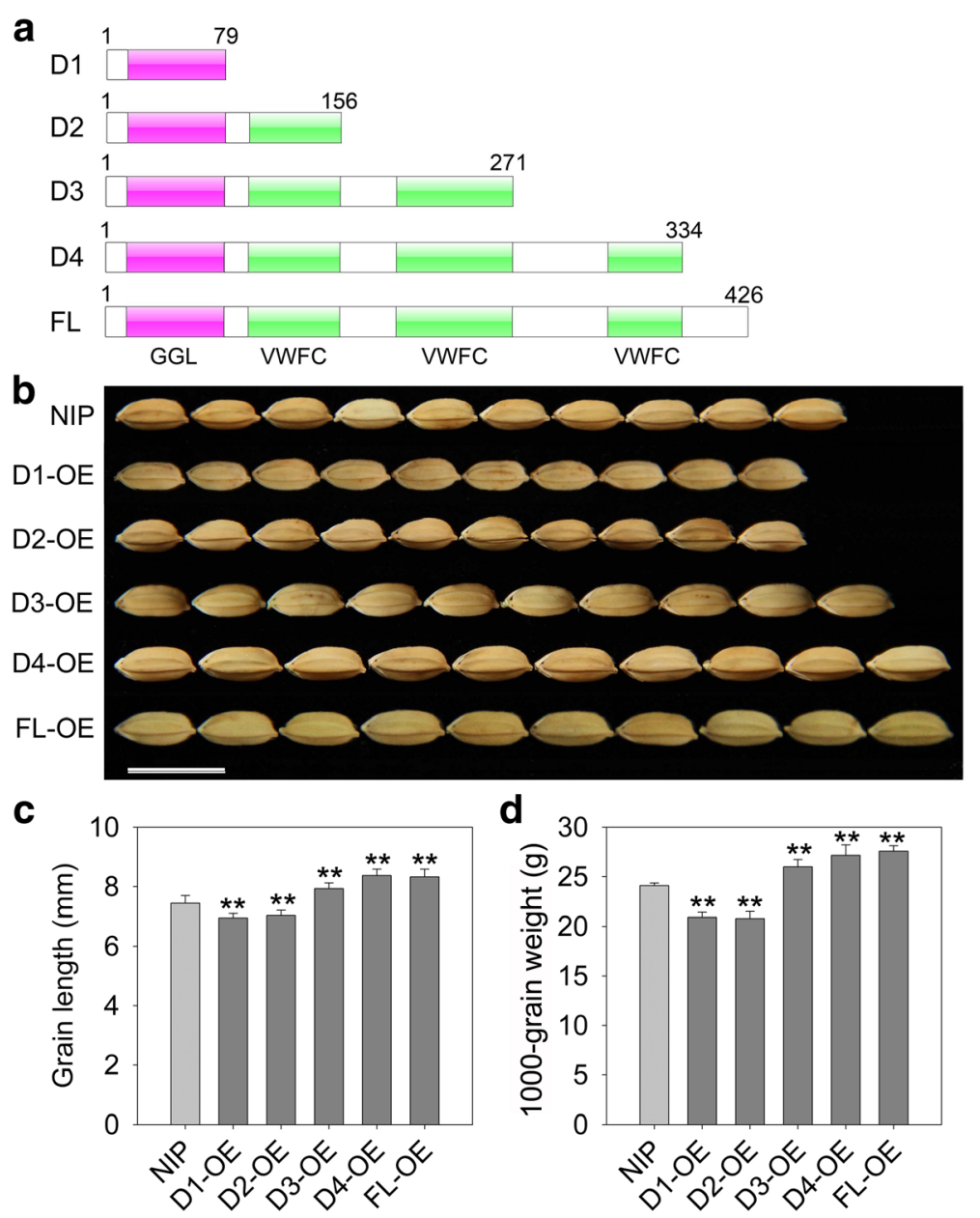

Fig. 3 Overexpression of different domains of qPE9-1 generated grains of different lengths and weights. a Several constructs covering different domains of QPE9-1. D1 contained the GGL domain and lacked three WWFC domains. D2, D3, and D4 contained the GGL domain and different numbers of WWFC domains. FL, full-length qPE9-1 protein. $\mathbf{b}$ Comparisons of grains from wild-type and transgenic plants carrying different constructs. Bar $=1 \mathrm{~cm}$. $\mathbf{c}$ Comparison of grain length between wild-type and overexpressing transgenic plants. $\mathbf{d}$ Comparison of 1000-grain weight between wild-type and overexpressing transgenic plants. Data are the average of two independent transgenic lines and are shown as means \pm SDs $(n=30)$. Student's t-test: ${ }^{* *} P<0.01$

WXG9-OE3 plants also had elongated panicle lengths and increased panicle sizes (Fig. $4 \mathrm{~b}$, e, and f). The panicle number per plant of overexpression lines was slightly decreased, but the difference was not significant (Fig. 4g). Additionally, the transgenic lines exhibited an obviously increased grain size (Fig. 4a, h, i, and j), and the 1000-grain weight of the three lines was increased by $23.27 \%, 24.46 \%$, and $24.57 \%$, respectively (Fig. $4 \mathrm{k}$ ). Thus, the grain yield per plant increased by $9.90 \%, 12.19 \%$, and $16.35 \%$, respectively, compared with wild-type (Fig. 41 ). These data suggest that $q P E 9-1$ promotes plant growth and improves grain yield per plant in an existing high-yield variety background.

\section{Discussion}

Heterotrimeric G proteins are universal signaling components in eukaryotes, and their regulatory properties have been thoroughly investigated in animals (Urano et al., 2012; Urano et al., 2013; Bender and Zipfel, 2018). The classic heterotrimers consist of three different subunits, designated $G_{\alpha}, G_{\beta}$, and $G_{\gamma}$. In mammalian cells, activation of G-protein-coupled receptors (GPCRs) upon ligand binding leads to GDP/GTP exchange and the activation of $G_{\alpha}$, which causes its dissociation from the $G_{\beta \gamma}$ dimer. Disassociated $G_{\alpha}$ and $G_{\beta \gamma}$ then activate their own downstream signaling elements to regulate various biological processes. The situation differs in plants, where the canonical $G_{\alpha}, G_{\beta}$, and $G_{\gamma}$ are encoded by a small number of genes (Bender and Zipfel, 2018). Because of the spontaneous guanine nucleotide exchange activity of plant $G_{\alpha}$ subunits and the absence of plant GPCRs, the molecular mechanism of heterotrimeric $G$ proteins for plant growth and development departs from the accepted animal paradigm (Trusov and Botella, 2016). 


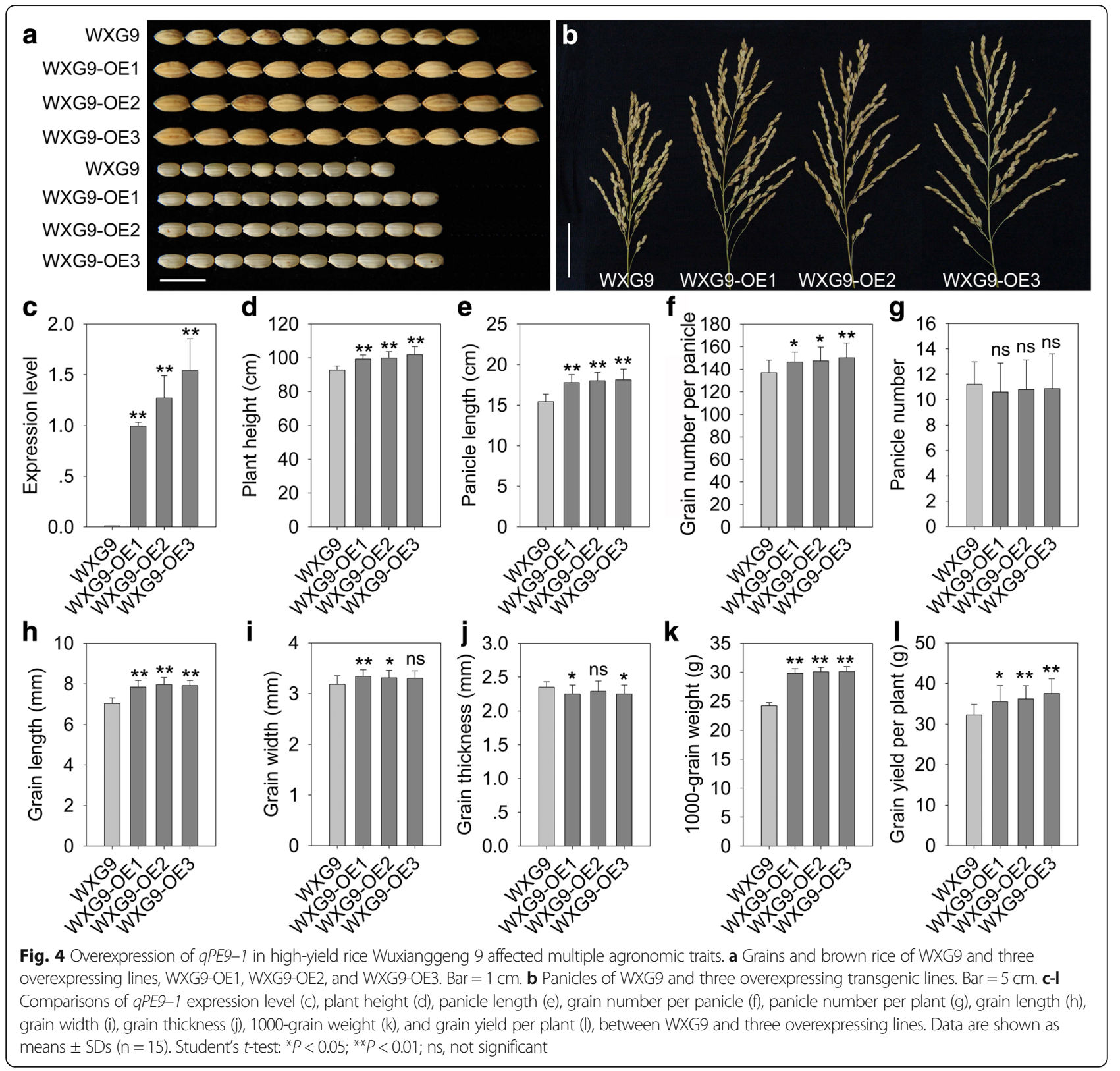

The function of $G_{\gamma}$ was initially thought to be restricted to anchoring $G_{\beta \gamma}$ dimers to the membrane. In plants, $G_{\gamma}$ subunits can be divided into three clades, type $A$, type $B$, and type $C$, which exhibit extraordinary structural diversities and differences compared with their mammalian counterparts (Xu et al., 2016). Type A $G_{\gamma}$ subunits are very small (fewer than 100 amino acids), but contain all the conserved features of mammalian $G_{\gamma}$ proteins. Type $B G_{\gamma}$ subunits lack the CaaX motif for isoprenylation (Trusov. et al., 2012; Urano et al., 2012). Type $C G_{\gamma}$ subunits belong to a novel class that is widespread throughout the plant kingdom but is nonexistent in animals (Sun et al., 2014). They have an N-terminal GGL domain, a weakly predicted transmembrane helix in the central region, and a long C-terminal cysteine-rich region that consists of VWFC and TNFR domains. In rice, the three homologs, GS3, qPE9-1/DEP1, and GGC2, belong to type C. Mao et al. (2010) showed that the OSR (GGL) domain of GS3 is both necessary and sufficient to function as a negative regulator. Loss-of-function of OSR results in long grains, while C-terminal TNFR/NGFR and VWFC domains have an inhibitory effect on the OSR function. However, the roles of each domain of qPE9-1/ DEP1 remained unclear.

In this study, we generated several homozygous knockout mutants of $q P E 9-1$ in the Nipponbare background using CRISPR/Cas9. All three independent mutants, qpe9-1\#1, qpe9-1\#2, and qpe9-1\#3, lost most of the 
qPE9-1 protein and showed a decreased grain length and weight (Fig. 2). We also obtained $q P E 9-1$-overexpressing lines in Nipponbare and Wuxianggeng 9 backgrounds, which all produced larger and heavy grains. These results confirmed that $q P E 9-1$ is functional and plays a positive role in rice grain length and weight. Recently, Sun et al. (2018) reported that DEP1 and GGC2, either individually or in combination, increase grain length when complexed with RGB1, which is consistent with our results. We further generated a series of transgenic lines overexpressing truncated qPE9-1 segments covering various protein domains. As shown in Fig. 3, the transformants overexpressing D1 and D2 constructs produced shorter and lighter grains, while those overexpressing D3 and D4 constructs had longer and heavier grains. The grain length of D4-OE plants was similar to that in transformants overexpressing full-length $q P E 9-1$. This indicated that the $\mathrm{N}$-terminal GGL of qPE9-1 negatively regulates rice grain length and weight, and that its inhibitory effect is suppressed by the C-terminal VWFC domains. Elevated levels of truncated $\mathrm{qPE} 9-1$ protein containing two or three VWFC domains were shown to increase grain length and weight, while only one VWFC domain was insufficient to modify the inhibition of the GGL domain on grain size. This suggested that the cysteine-rich C-terminal tail of qPE9-1 is critical in the regulation of grain length and weight.

The N-terminal GGL and C-terminal VWFC domains of qPE9-1 exert antagonistic effects in grain length and weight regulation, which is similar to the head-to-tail model obtained from GS3 (Mao et al., 2010). Sun et al. (2018) further proposed a genetic model depicting the pathway of the $G$ proteins in grain size regulation. In this model, qPE9-1/DEP1 and GGC2 positively regulate grain size, while GS3 alone has no effect. However, the competitive interaction of GS3 with RGB1 disrupts RGB1-qPE9-1/DEP1 and RGB1-GGC2 dimers, resulting in short grains. This model also provides a possible explanation for the fact that the overexpressed GGL domain of qPE9-1 decreased the grain length and weight in this study. However, it was also demonstrated that both GS3 and qPE9-1/DEP1 play negative roles in the regulation of grain size by promoting activity of the OsMADS1 transcription factor (Liu et al., 2018). Previously, Li et al. (2016a) assessed the genetic effects of qPE9-1/DEP1 using CRISPR/Cas9, and found that the grains of two knockout mutants were significantly lighter than those of wild-type plants. Taken together, we believe that $q P E 9-1 / D E P 1$ is a positive regulator of rice grain length and weight.

Suppression of $R G B 1$ expression causes dwarfism and small grains (Utsunomiya et al., 2011), so RGB1 is also considered a positive regulator of rice grain size. RGB1 forms a dimer with qPE9-1/DEP1 and GGC2 to promote grain growth (Sun et al., 2018). However, RGB1 mutants have not been identified in rice, and it is generally agreed that $R G B 1$ knockout is lethal. We obtained several transgenic lines of $R G B 1$ in Nipponbare (carrying the $q P E 9-1 / D E P 1$ allele) and Wuyungeng 8 (carrying the qpe9-1/dep1 allele) backgrounds, using RNA interference-mediated gene silencing, which resulted in decreased plant height and reduced grain size (data not shown). It was recently reported that $R G B 1$ overexpression led to small grains (Liu et al., 2018). However, we observed no obvious phenotypic variation in grain size in Nipponbare plants overexpressing $R G B 1$ (data not shown). The actual function of $R G B 1$ in rice grain size regulation is therefore unclear, although it is possible that RGB1 protein in wild-type is sufficient to form a dimer with $\mathrm{G}_{\beta \gamma}$ to regulate plant and grain growth.

In the present study, we also overexpressed $q P E 9-1$ in a high-yield rice variety, WXG9, which carries qpe9-1 and produces short and erect panicles. As expected, homozygous and stable transgenic lines exhibited increased plant height and panicle length, as well as enlarged grain size (Fig. 4). The grain yield per plant of the overexpression lines was significantly increased compared with WXG9, although the panicle number per plant was slightly decreased. However, during 20092016, we observed frequent lodging in $q P E 9-1$-overexpressing lines in WXG9, especially in conditions of high nitrogen input and high plant density (data not shown). The introgression of $q P E 9-1$ is known to significantly increase plant height (Zhou et al., 2009; Yi et al., 2011). Because plant height is negatively associated with the rice anti-lodging trait, the loss-of-function qpe9-1 allele appears to enhance the rice anti-lodging capacity by reducing plant height.

The genetic effects of $q P E 9-1 / D E P 1$ on grain yield are controversial. Huang et al. (2009) indicated that the mutation allele qpe9-1/dep1 increased grain yield per plant. However, several studies reported that qpe9-1 has negative or background-dependent roles on grain yield per plant (Chen et al., 2006; Zhou et al., 2009; Fumio et al., 2011; Yi et al., 2011; Lu et al., 2013). In fact, the qpe9-1/ dep 1 allele is only used in japonica varieties, while the qPE9-1/DEP1 allele is widely employed in indica varieties. It remains unclear why a gene that decreases grain yield per plant is widely used in japonica rice production. Previously, we proposed that qpe9-1 is a double-edged sword allele in rice breeding, in that it shapes an ideal plant architecture but has negative effects on the individual yield of the plant (Zhou et al., 2009). Rice varieties carrying qpe9-1 usually produce short, erect panicles and leaves. This compact plant architecture is not only beneficial in improving ventilation and light penetration, but is also suitable for close planting. In the present study, 
WXG9 overexpression lines produced larger panicle sizes compared with wild-type (Fig. 4b, f). Previously, we reported that $q P E 9-1$ had no effect on the grain number per panicle (Zhou et al., 2009), although Huang et al. (2009) indicated that the qpe9-1/dep1 allele significantly increased grain number per panicle. Moreover, a loss-of-function mutation of DENSE PANICLE 1, which is allelic to $q P E 9-1 / D E P 1$, caused semi-dwarfness and a slightly increased number of spikelets (Fumio et al., 2011), while the deletion of $q P E 9-1 / D E P 1$ in indica rice via CRISPR/Cas9 resulted in small grains and reduced panicle sizes (Wang et al., 2017). Taken together, the genetic effects of qPE9-1/qpe9-1 appear to be complicated, and may change according to different genetic backgrounds and field conditions.

\section{Conclusions}

In the present study, we generated a series of transgenic plants expressing different truncated $\mathrm{qPE} 9-1$ protein constructs through constitutive expression and CRISPR/ Cas9 strategies. Phenotypic analysis indicated that full-length or long-tailed qPE9-1 produced long and heavy grains, while the GGL domain alone and short-tailed qPE9-1 produced short and light grains. Moreover, the long C-terminus of qPE9-1 including two or three C-terminal VWFC domains effectively repressed the negative effects of the GGL domain on grain length and weight. Manipulation of the C-terminal length of qPE9-1 through genetic engineering can therefore be used to generate varieties with various grain lengths and weights according to different requirements in rice breeding. Overexpression of $q P E 9-1$ increased grain size and yield in the Wuxianggeng 9 background, but led to lodging in some years. Breeders should take into account other factors including genetic backgrounds and planting conditions in the use of $q P E 9-1 / q p e 9-1$.

\section{Methods}

\section{Vector construction and transformation}

Two specific target guide RNA sequences located in the second and fifth exons were selected to generate $q P E 9-1$ mutants. The final fragment was inserted into a CRISPR/Cas9 system (Baige Biotech, Hangzhou City, China) in which the Cas9 destination vector was driven by the maize ubiquitin promoter for expression in rice, and sgRNA expression was driven by the U6 promoter. The CRISPR/Cas9 construct was transformed into Nipponbare via Agrobacterium tumefaciens-mediated transformation. The different variations of $q P E 9-1$ obtained from CRISPR/Cas9 editing were sequenced with specific primers (Table S1). The homozygous transgenic lines of mutants were used for functional analysis.

The full and partial coding regions of $q P E 9-1$ were amplified from Nipponbare cDNA, and inserted into the
p1301UbiNOS vector to generate several overexpression constructs which were driven by a constitutively expressed maize ubiquitin promoter (Zhou et al., 2009). All constructs were transferred into Nipponbare and Wuxianggeng 9 by $A$. tumefaciens-mediated transformation.

\section{Plant growth conditions}

Forty plants of each line were grown in the experimental field of Yangzhou University $\left(\mathrm{E} 119^{\circ} 25^{\prime} / \mathrm{N} 32^{\circ} 23^{\prime}\right)$ in the summer of 2017 for molecular and phenotypic evaluation. Ten seedlings (approximately 4-weeks-old) per row were transplanted with a distance of $17.0 \mathrm{~cm}$ between plants and $23.3 \mathrm{~cm}$ between rows. After transplanting, they were fertilized with nitrogen ( $225 \mathrm{~kg} \mathrm{ha}^{-1}$ as urea), phosphorus (50 kg ha ${ }^{-1}$ as single superphosphate), and potassium (60 $\mathrm{kg} \mathrm{ha}^{-1}$ as $\mathrm{KCl}$ ). Field management and disease and pest control followed standard procedures to prevent yield loss during the growth period.

\section{Phenotypic evaluation}

At maturity, several traits of wild-type and transgenic lines were measured to investigate the roles of each domain of qPE9-1. Plant height was measured from the ground surface to the tallest panicle. Panicles of the main stem were selected for measuring panicle length and counting the grain number per panicle. Panicle number per plant was the number of effective panicles with 10 or more grains. The length, width, and thickness of grains were measured using vernier calipers after harvesting and storage at $37^{\circ} \mathrm{C}$ for at least 2 weeks. The weight of 100 plump grains was obtained and then converted to the 1000-grain weight. All grains of one plant were weighed to measure the grain yield per plant.

\section{RNA preparation and reverse transcription quantitative PCR (qPCR)}

Total RNA was extracted with an RNA Prep Pure Kit (Tiangen Biotech, Beijing City, China) in accordance with the manufacturer's instructions and then treated with DNase to digest any genomic DNA. cDNA was synthesized from $1 \mu \mathrm{g}$ of total RNA using a reverse transcription kit (Tiangen Biotech).

qPCR was performed in a total volume of $25 \mu \mathrm{l}$, which consisted of $2 \mu \mathrm{l}$ of cDNA, $0.2 \mathrm{mM}$ of each primer, and $12.5 \mu \mathrm{l}$ of $2 \times$ SYBR Green PCR Master Mix (Takara Bio, Shiga, Japan). The qPCR assay was conducted using a qPCR system (ViiA7, Applied Biosystems, Foster City, USA) using the following program: $95^{\circ} \mathrm{C}$ for $3 \mathrm{~min}$ followed by 40 cycles of $94^{\circ} \mathrm{C}$ for $30 \mathrm{~s}, 55^{\circ} \mathrm{C}$ for $30 \mathrm{~s}$, and $72{ }^{\circ} \mathrm{C}$ for $40 \mathrm{~s}$. qPCR analysis was carried out using the rice actin gene (LOC_Os03g50885) as an internal control. Data are presented as the mean values of three replicates. Relative gene expression was calculated using the 
$2^{-\Delta \Delta C T}$ method. $\mathrm{qPCR}$ primers are listed in Additional file 1: Table S1.

\section{Statistical analysis}

All numerical data are presented as the means \pm SDs (error bars indicate the standard deviations of the means). Statistical analyses were carried out using Excel (Microsoft, USA) and SigmaPlot software (Systat, USA). The differences between transgenic and wild-type plants were determined using the Student's $t$-test $\left({ }^{*}, P<0.05\right.$; **, $P<0.01 ;$ ns, not significant).

\section{Additional file}

Additional file 1: Table S1. Primers used in this study. (DOC $32 \mathrm{~kb}$ )

\section{Acknowledgments}

We thank Dr. Dongping Zhang (Yangzhou University) for his helpfu suggestions on how to improve this work.

\section{Abbreviations}

CRISPR/Cas9: clustered regularly interspaced short palindromic repeats and CRISPR-associated protein 9; qPCR: quantitative PCR; QTL: quantitative trait locus; GGL, G gamma-like domain; OSR, organ size regulation; TNFR/NGFR, tumor necrosis factor receptor/nerve growth factor receptor; WWFC, von Willebrand factor type C; GPCRs, G-protein-coupled receptors.

\section{Funding}

This work was supported by grants from the National Training Programs of Innovation and Entrepreneurship for Undergraduates, the Natural Science Fund for Colleges and Universities in Jiangsu Province (17KJA210002), and the Priority Academic Program Development of Jiangsu Higher Education Institutions.

\section{Availability of data and materials}

All data supporting the conclusions of this article are provided within the article (and its additional files)

\section{Authors' contributions}

$Y Z$ and $G L$ conceived and designed the experiments. $X L, Q T$, and $Y Z$ conducted most of the experiments. $Z Y$ analyzed the data. $X L$ and $Y Z$ wrote the manuscript. All authors reviewed and approved the manuscript.

\section{Ethics approval and consent to participate}

Not applicable.

\section{Consent for publication}

Not applicable.

\section{Competing interests}

The authors declare that they have no competing interests.

\section{Publisher's Note}

Springer Nature remains neutral with regard to jurisdictional claims in published maps and institutional affiliations.

\section{Author details}

1Jiangsu Key Laboratory of Crop Genetics and Physiology / Key Laboratory of Plant Functional Genomics of the Ministry of Education / Jiangsu Key Laboratory of Crop Genomics and Molecular Breeding, Agricultural College of Yangzhou University, Yangzhou 225009, China. ${ }^{2}$ Jiangsu Co-Innovation Center for Modern Production Technology of Grain Crops, Yangzhou University, Yangzhou 225009, China.
Received: 2 October 2018 Accepted: 6 January 2019

Published online: 31 January 2019

\section{References}

Ashikari M, Wu JZ, Yano M, Sasaki T, Yoshimura A (1999) Rice gibberellininsensitive dwarf mutant gene Dwarf 1 encodes the alpha-subunit of GTPbinding protein. Proc Natl Acad Sci U S A 96(18):10284-10289

Bender KW, Zipfel C (2018) Plant G-protein activation: connecting to plant receptor kinases. Cell Res 28(7):697-698

Chakravorty D, Trusov Y, Zhang W, Acharya BR, Sheahan MB, McCurdy DW, Assmann SM, Botella JR (2011) An atypical heterotrimeric G-protein gammasubunit is involved in guard cell $\mathrm{K}\left({ }^{+}\right)$-channel regulation and morphological development in Arabidopsis thaliana. Plant J 67(5):840-851

Chen JG, Willard FS, Huang J, Liang JS, Chasse SA, Jones AM, Siderovski DP (2003) A seven-transmembrane RGS protein that modulates plant cell proliferation. Science 301(5640):1728-1731

Chen XG, Liu JB, Hong DL (2006) Genetic analysis on panicle angle and number of spikelets per panicle by using six generations of three crosses derived from erectxcurve panicles in japonica rice (Oryza sativa L.). Acta Agron Sin 32(8): 1143-1150

Fageria NK (2007) Yield physiology of rice. J of Plant Nutr 30(4-6):843-879

Fan CC, Xing YZ, Mao HL, Lu TT, Han B, Xu CG, Li XH, Zhang QF (2006) GS3, a major QTL for grain length and weight and minor QTL for grain width and thickness in rice, encodes a putative transmembrane protein. Theor Appl Genet 112(6):1164-1171

Ferrero-Serrano A, Su Z, Assmann SM (2018) Illuminating the role of the Ga heterotrimeric $\mathrm{G}$ protein subunit, $\mathrm{RGA1}$, in regulating photoprotection and photoavoidance in rice. Plant Cell Environ 41(2):451-468

Fitzgerald MA, McCouch SR, Hall RD (2009) Not just a grain of rice: the quest for quality. Trends Plant Sci 14(3):133-139

Fujisawa Y, Kato T, Ohki S, Ishikawa A, Kitano H, Sasaki T, Asahi T, Iwasaki Y (1999) Suppression of the heterotrimeric $\mathrm{G}$ protein causes abnormal morphology, including dwarfism, in rice. Proc Natl Acad Sci U S A 96(13):7575-7580

Fumio T-S, Yasushi K, Hiroshi K, Haruko O, Akemi T, Naho H, Akio M, Hirohiko H, Hidemi K, Masahiro Y, Seiichi T (2011) A loss-of-function mutation of rice DENSE PANICLE 1 causes semi-dwarfness and slightly increased number of spikelets. Breed Sci 61(1):17-25

Hu X, Wang C, Fu Y, Liu Q, Jiao XZ, Wang K (2016) Expanding the range of CRISPR/Cas9 genome editing in rice. Mol Plant 9(6):943-945

Huang XZ, Qian Q, Liu ZB, Sun HY, He SY, Luo D, Xia GM, Chu CC, Li JY, Fu XD (2009) Natural variation at the DEP1 locus enhances grain yield in rice. Nat Genet 41(4):494-497

Ishida T, Tabata R, Yamada M, Aida M, Mitsumasu K, Fujiwara M, Yamaguchi K, Shigenobu S, Higuchi M, Tsuji H, Shimamoto K, Hasebe M, Fukuda H, Sawa S (2014) Heterotrimeric $\mathrm{G}$ proteins control stem cell proliferation through CLAVATA signaling in Arabidopsis. EMBO Rep 15(11):1202-1209

Kaur J, Roy Choudhury S, Vijayakumar A, Hovis L, Rhodes Z, Polzin R, Blumenthal D. Pandey $S$ (2018) Arabidopsis type III g gamma protein AGG3 is a positive regulator of yield and stress responses in the model monocot Setaria viridis. Front Plant Sci 9:109

Li JM, Thomson M, McCouch SR (2004) Fine mapping of a grain-weight quantitative trait locus in the pericentromeric region of rice chromosome 3. Genetics 168(4):2187-2195

Li JY, Sun YW, Du JL, Zhao YD, Xia LQ (2017) Generation of targeted point mutations in rice by a modified CRISPR/Cas9 system. Mol Plant 10(3):526-529

Li MR, Li XX, Zhou ZJ, Wu PZ, Fang MC, Pan XP, Lin QP, Luo WB, Wu GJ, Li HQ (2016a) Reassessment of the four yield-related genes Gn1a, DEP1, GS3, and IPA1 in rice using a CRISPR/Cas9 system

Li N, Li YH (2016b) Signaling pathways of seed size control in plants. Curr Opin Plant Biol 33:23-32

Li N, Xu R, Duan PG, Li YH (2018) Control of grain size in rice. Plant Reprod 31(3): 237-251

Li SJ, Liu YJ, Zheng LY, Chen LL, Li N, Corke F, Lu YR, Fu XD, Zhu ZG, Bevan MW, $\mathrm{Li}$ YH (2012) The plant-specific G protein $\gamma$ subunit AGG3 influences organ size and shape in Arabidopsis thaliana. New Phytol 194(3):690-703

Liu Q, Han RX, Wu K, Zhang JQ, Ye YF, Wang SS, Chen JF, Pan YJ, Li Q, Xu XP, Zhou JW, Tao DY, Wu YJ, Fu XD (2018) G-protein $\beta$ Y subunits determine grain size through interaction with MADS-domain transcription factors in rice. Nat Commun 9(1):852

Lu Y, Zhu JK (2017) Precise editing of a target base in the rice genome using a modified CRISPR/Cas9 system. Mol Plant 10(3):523-525 
Lu ZF, Yu H, Xiong GS, Wang J, Jiao YQ, Liu GF, Jing YH, Meng XB, Hu XM, Qian Q, Fu XD, Wang YH, Li JY (2013) Genome-wide binding analysis of the transcription activator ideal plant architecture1 reveals a complex network regulating rice plant architecture. Plant Cell 25(10):3743-3759

Ma XL, Zhu QL, Chen YL, Liu YG (2016) CRISPR/Cas9 platforms for genome editing in plants: developments and applications. Mol Plant 9(7):961-974

Ma Y, Dai XY, Xu YY, Luo W, Zheng XM, Zeng DL, Pan YJ, Lin XL, Liu HH, Zhang DJ, Xiao J, Guo XY, Xu SJ, Niu YD, Jin JB, Zhang H, Xu X, Li LG, Wang W, Qian Q, Ge S, Chong K (2015) COLD1 confers chilling tolerance in rice. Cell 160(6): 1209-1221

Mao HL, Sun SY, Yao JL, Wang CR, Yu SB, Xu CG, Li XH, Zhang QF (2010) Linking differential domain functions of the GS3 protein to natural variation of grain size in rice. Proc Natl Acad Sci U S A 107(45):19579-19584

Peng YC, Chen LL, Li SJ, Zhang YY, Xu R, Liu ZP, Liu WX, Kong JJ, Huang XH, Wang YC, Cheng BJ, Zheng LY, Li YH (2018) BRI1 and BAK1 interact with G proteins and regulate sugar-responsive growth and development in Arabidopsis. Nat Commun 9(1):1522

Shi CY, Qi C, Ren HY, Huang AX, Hei SM, She XP (2015) Ethylene mediates brassinosteroid-induced stomatal closure via Ga protein-activated hydrogen peroxide and nitric oxide production in Arabidopsis. Plant J 82(2):280-301

Subramaniam G, Trusov Y, Lopez-Encina C, Hayashi S, Batley J, Botella JR (2016) Type $B$ heterotrimeric $G$ protein $\gamma$-subunit regulates auxin and $A B A$ signaling in tomato. Plant Physiol 170(2):1117-1134

Sun HY, Qian Q, Wu K, Luo JJ, Wang SS, Zhang CW, Ma YF, Liu Q, Huang XZ, Yuan QB, Han RX, Zhao M, Dong GJ, Guo LB, Zhu XD, Gou ZH, Wang W, Wu YJ, Lin HX, Fu XD (2014) Heterotrimeric G proteins regulate nitrogen-use efficiency in rice. Nat Genet 46(6):652-656

Sun SY, Wang L, Mao HL, Shao L, Li XH, Xiao JH, Ouyang YD, Zhang QF (2018) A G-protein pathway determines grain size in rice. Nat Commun 9(1):851

Tan YF, Xing YZ, Li JX, Yu SB, Xu CG, Zhang QF (2000) Genetic bases of appearance quality of rice grains in Shanyou 63, an elite rice hybrid. Theor Appl Genet 101(5-6):823-829

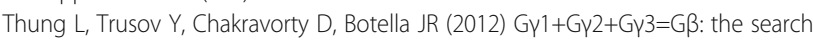
for heterotrimeric G-protein $\gamma$ subunits in Arabidopsis is over. J Plant Physiol 169(5):542-545

Trusov Y, Botella JR (2016) Plant G-proteins come of age: breaking the bond with animal models. Front Chem 4:24

Trusov Y, Chakravorty D, Botella JR (2012) Diversity of heterotrimeric G-protein Y subunits in plants. BMC Res Nots 5:608

Ueguchi-Tanaka M, Fujisawa Y, Kobayashi M, Ashikari M, Iwasaki Y, Kitano H, Matsuoka M (2000) Rice dwarf mutant d1, which is defective in the alpha subunit of the heterotrimeric $\mathrm{G}$ protein, affects gibberellin signal transduction. Proc Natl Acad Sci U S A 97(21):11638-11643

Ullah H, Chen JG, Young JC, Im KH, Sussman MR, Jones AM (2001) Modulation of cell proliferation by heterotrimeric $\mathrm{G}$ protein in Arabidopsis. Science 292(5524):2066-2069

Urano D, Chen JG, Botella JR, Jones AM (2013) Heterotrimeric G protein signalling in the plant kingdom. Open Biol 3(3):120186

Urano D, Jones JC, Wang H, Matthews M, Bradford W, Bennetzen JL, Jones AM (2012) G protein activation without a GEF in the plant kingdom. PLoS Genet 8(6):e1002756

Utsunomiya Y, Samejima C, Takayanagi Y, Izawa Y, Yoshida T, Sawada Y, Fujisawa $Y$, Kato $H$, Iwasaki $Y$ (2011) Suppression of the rice heterotrimeric $G$ protein $\beta$-subunit gene, RGB1, causes dwarfism and browning of internodes and lamina joint regions. Plant J 67(5):907-916

Wang C, Shen L, Fu YP, Yan CJ, Wang KJ (2015) A simple CRISPR/Cas9 system for multiplex genome editing in rice. J Genet Genomic 42(12):703-706

Wang Y, Geng LZ, Yuan ML, Wei J, Jin C, Li M, Yu K, Zhang Y, Jin HB, Wang E, Chai ZJ, Fu XD, Li XG (2017) Deletion of a target gene in Indica rice via CRISPR/Cas9. Plant Cell Rep 36(8):1333-1343

Wettschureck N, Offermanns S (2005) Mammalian G proteins and their cell type specific functions. Physiol Rev 85(4):1159-1204

Xu Q, Zhao MZ, Wu K, Fu XD, Liu Q (2016) Emerging insights into heterotrimeric G protein signaling in plants. J Genet Genomic 43(8):495-502

Yang JC, Zhang JH (2010) Grain-filling problem in 'super' rice. J Exp Bot 61(1):1-5

Yi XH, Zhang ZJ, Zeng SY, Tian CY, Peng JC, Li M, Lu Y, Meng QC, Gu MH, Yan CJ (2011) Introgression of aPE9-1 allele, conferring the panicle erectness, leads to the decrease of grain yield per plant in japonica rice (Oryza sativa L.). J Genet Genomic 38(5):217-223
Yu Y, Assmann SM (2015) The heterotrimeric G-protein $\beta$ subunit, AGB1, plays multiple roles in the Arabidopsis salinity response. Plant Cell Environ 38(10): 2143-2156

Zhang DP, Zhou Y, Yin JF, Yan XJ, Lin S, Xu WF, Baluska F, Wang YP, Xia YJ, Liang GH, Liang JS (2015) Rice G-protein subunits qPE9-1 and RGB1 play distinct roles in abscisic acid responses and drought adaptation. J Exp Bot 66(20): $6371-6384$

Zhang T, Xu P, Wang W, Wang S, Caruana JC, Yang HQ, Lian H (2017) Arabidopsis G-protein $\beta$ subunit AGB1 interacts with BES1 to regulate brassinosteroid signaling and cell elongation. Front Plant Sci 8:2225

Zhou Y, Zhu JY, Li ZY, Yi CD, Liu J, Zhang HG, Tang SZ, Gu MH, Liang GH (2009) Deletion in a quantitative trait gene QPE9-1 associated with panicle erectness improves plant architecture during rice domestication. Genetics 183(1):315-324

\section{Submit your manuscript to a SpringerOpen ${ }^{\circ}$ journal and benefit from:}

- Convenient online submission

- Rigorous peer review

- Open access: articles freely available online

- High visibility within the field

- Retaining the copyright to your article

Submit your next manuscript at $\boldsymbol{\nabla}$ springeropen.com 